EPJ Web of Conferences 59, 09006 (2013)

DOI: $10.1051 /$ epjconf/20135909006

(C) Owned by the authors, published by EDP Sciences, 2013

\title{
Modeling warm dense matter experiments using the 3D ALE-AMR code and the move toward exascale computing
}

\author{
Alice Koniges ${ }^{1, a}$, Wangyi Liu ${ }^{1}$, John Barnard ${ }^{2}$, Alex Friedman², Grant Logan ${ }^{1}$, \\ David Eder ${ }^{2}$, Aaron Fisher ${ }^{2}$, Nathan Masters ${ }^{2}$ and Andrea Bertozzi ${ }^{3}$ \\ ${ }^{1}$ Lawrence Berkeley National Laboratory \\ ${ }^{2}$ Lawrence Livermore National Laboratory \\ ${ }^{3}$ University of California, Los Angeles
}

\begin{abstract}
The Neutralized Drift Compression Experiment II (NDCX II) is an induction accelerator planned for initial commissioning in 2012. The final design calls for a $3 \mathrm{MeV}, \mathrm{Li}+$ ion beam, delivered in a bunch with characteristic pulse duration of $1 \mathrm{~ns}$, and transverse dimension of order $1 \mathrm{~mm}$. The NDCX II will be used in studies of material in the warm dense matter (WDM) regime, and ion beam/hydrodynamic coupling experiments relevant to heavy ion based inertial fusion energy. We discuss recent efforts to adapt the 3D ALE-AMR code to model WDM experiments on NDCX II. The code, which combines Arbitrary Lagrangian Eulerian (ALE) hydrodynamics with Adaptive Mesh Refinement (AMR), has physics models that include ion deposition, radiation hydrodynamics, thermal diffusion, anisotropic material strength with material time history, and advanced models for fragmentation. Experiments at NDCX-II will explore the process of bubble and droplet formation (two-phase expansion) of superheated metal solids using ion beams. Experiments at higher temperatures will explore equation of state and heavy ion fusion beamto-target energy coupling efficiency. Ion beams allow precise control of local beam energy deposition providing uniform volumetric heating on a timescale shorter than that of hydrodynamic expansion. We also briefly discuss the effects of the move to exascale computing and related computational changes on general modeling codes in fusion.
\end{abstract}

\section{INTRODUCTION}

Warm Dense Matter (WDM) is an emerging and challenging field that is at the crossroads of strongly and weakly coupled plasmas, degenerate and non-degenerate matter, and solid, liquid and vapor states. The basic physical properties, e.g., opacities, conductivities, dielectric functions, heat capacities, and phase transitions, are not well understood for WDM conditions. The response of material to dynamic loading, e.g., droplet and bubble formation, is of fundamental interest and depends critically on the controlling physical parameters. The experimental challenge is to create sufficiently warm $(0.5-5 \mathrm{eV})$, nearly uniform matter at near solid densities. Our approach is to use a heating source that penetrates a sample, e.g., high-energy ions, with a pulse short enough to achieve heating prior to significant hydrodynamic motion. Accurate modeling of experiments is critical to obtain a true understanding of WDM properties and related phenomena. We are developing a 3D open-source, multi-physics, multimaterial code ALE-AMR, for modeling the WDM regime. Recently changes in computer architecture are forcing radical changes in computational modeling for the drive to exascale computing. In this paper, we also discuss the effects of these computational changes on large scale energy modeling codes such as ALE-AMR

\footnotetext{
${ }^{a}$ e-mail: aekoniges@lbl.gov
}

This is an Open Access article distributed under the terms of the Creative Commons Attribution License 2.0, which permits unrestricted use, distribution, and reproduction in any medium, provided the original work is properly cited. 


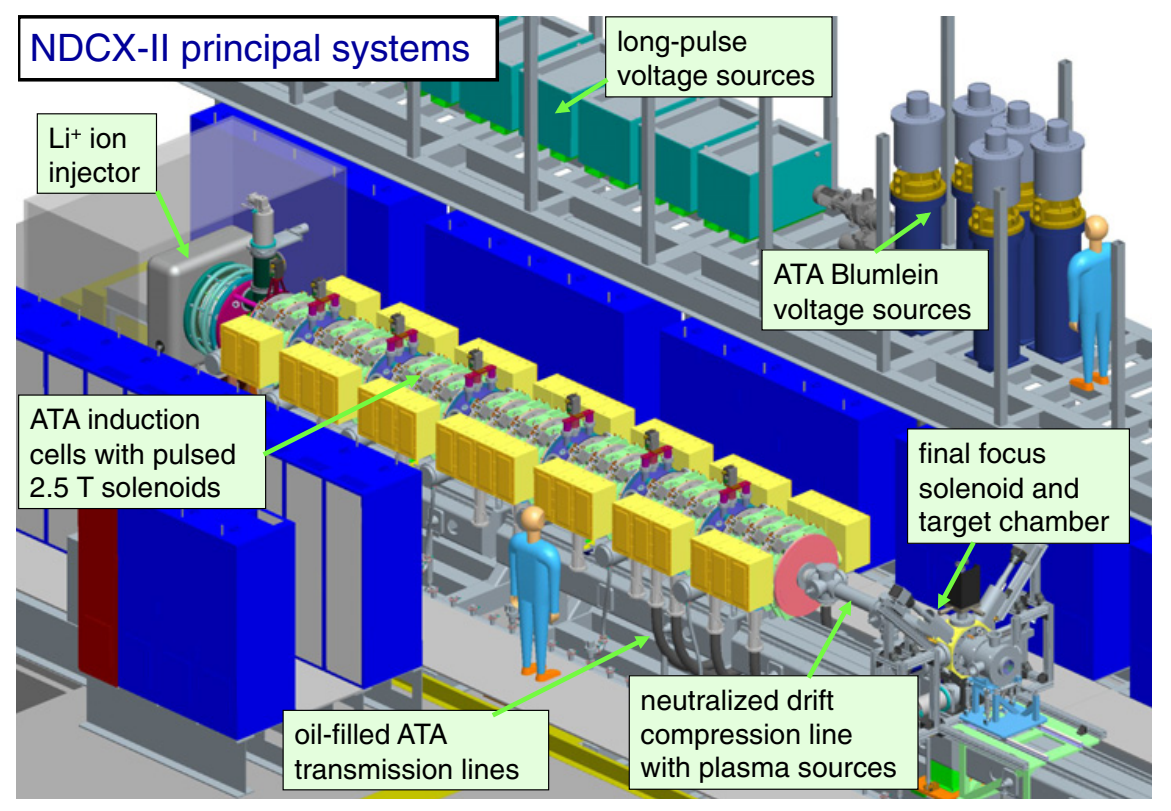

Figure 1. The NDCX-II machine at LBNL.

\section{THE NDCX-II FACILITY}

A key feature for NDCX-II is inflight longitudinal "drift compression" of the beam to achieve a high intensity pulse; this is a process analogous to chirped-pulse compression of a short-pulse laser beam. A head-to-tail velocity gradient is imparted to the ion beam by a set of induction cells (accelerating elements), and the pulse then compresses as it drifts down a beam line, in a neutralizing plasma environment which provides space-charge compensation. NDCX-II will accelerate a beam containing 20-50 $\mathrm{nC}$ of $\mathrm{Li}+$ ions to $1.2-3 \mathrm{MeV}$ and compress it into a sub-ns pulse with a 1-mm radius spot size. (Initial operation will have longer pulses and larger focal spots as discussed below.) The machine is an induction linac with custom voltage waveforms to control the longitudinal space charge forces and compress the pulse. Overall longitudinal compression factors of 500X are required to achieve a 1-ns pulse, but most of that compression occurs in the accelerator [1]. To be cost effective, NDCX-II reuses and modifies induction cores and cells and some of the pulsed power hardware from LLNL's decommissioned Advanced Test (electron) Accelerator (ATA). Up to 46 cells could be used on NDCX-II but the initial configuration will consist of 27 cells that reach an ion energy of $1.2 \mathrm{MeV}$. Figure 1 shows the layout of the NDCX-II facility. Extensive particle-in-cell computer simulation studies have enabled an attractive physics design that meets the stringent cost goal [1]. The blumleins (blue cylinders in the figure) can energize the ferrite cores in the induction cells with voltage pulses up to $250 \mathrm{kV}$ for $70 \mathrm{~ns}$. In order to minimize the accelerator length and to optimize the usage of pulsed power, an initial stage of non-neutral beam compression, at the first few induction cells after the injector, will shorten the pulse length from $500 \mathrm{~ns}$ to less than $70 \mathrm{~ns}$. Long-pulse voltage generators are used at the front end while blumleins power the rest of the acceleration. The shorter pulse (sub-ns) and longer ion range as compared to NDCX-I will allow more nearly isochoric heating of the target.

\section{MODELING WORK}

Simulations of NDCX-II targets require a large number of models/packages to capture the essential physics. The ALE-AMR code has ion, x-ray and laser deposition, radiation hydrodynamics, 

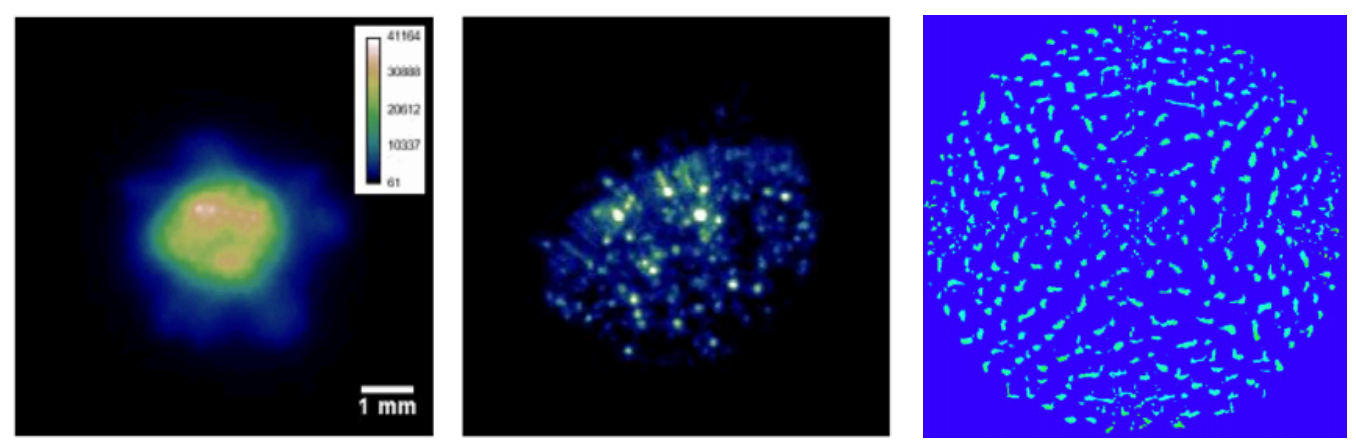

Figure 2. (left) Gated camera image (gate width $=6 \mu \mathrm{s}$ ) of a carbon target heated by a NDCX-I beam. (center) Shower of hot platinum debris fragments/droplets $500 \mu$ s after the beam pulse. (right) Preliminary ALE-AMR simulation for a surface tension model in 2D with symmetry. The image shows an initially heated titanium droplet that expands due to having higher pressure than its surroundings, and then break up into droplets. Initial radius is $0.1 \mu \mathrm{m}$, and the simulation picture is $1 \mu \mathrm{m}$ by $1 \mu \mathrm{m}$.

thermal diffusion, anisotropic material strength with material time history, and advanced models for fragmentation including void insertion [2]. When solid material fails in the context of a material failure condition in the code, a small volume of void is inserted in the zone. If the zone is stretched due to tensile forces or a divergent velocity field, the majority of the volume increase in the zone is due to void growth. The interface reconstruction scheme allows for void regions in neighboring zones to merge and can produce fragments of material surrounded by void. We are exploring a similar approach for modeling breakup of droplets. A study of surface tension models in ALE-AMR using the advective Cahn-Hilliard equations has been described [3]. Droplets have been observed during NDCX-I experiments for a range of materials. Figure 2 shows gated images for carbon and platinum. An ALE-AMR simulation of a titanium droplet is given in the right image. While these are not images of the same material, there are basic physics of surface tension effects that are extremely difficult to capture mathematically. The point of these images is to show that the models for droplet break-up we are exploring have the capacity to break into droplets like we see in many of the experiments. Using two-dimensional symmetry for simplicity and a simplified the beam heating process with an initially-heated droplet, the image of the droplet breakup shows some visual similarity to the experimental results, which will lead us to important model-with-data comparisons when NDCX-II starts to produce data. For direct comparison with experiments, additional physics, e.g., beam deposition, and a significantly larger computational domain to match the experimental conditions are required. The surface tension model used here is the Kortweg model [4] whereby the standard fluid equations containing a stress tensor are augmented with a term on the RHS of the momentum equation containing a parameter $K$ that determines surface tension.

$$
\begin{gathered}
\frac{d \rho}{d t}=\nabla \cdot(\rho \mathbf{V}) \\
\rho \frac{d \mathbf{V}}{d t}+\mathbf{V} \cdot \nabla \mathbf{V}=-\nabla p+\nabla \cdot \sigma_{1}+K \nabla \cdot S \\
S=\left(\frac{1}{2}|\nabla \rho|^{2}+\rho \Delta \rho\right) I-\nabla \rho \otimes \nabla \rho .
\end{gathered}
$$




\section{EPJ Web of Conferences}

Although this model works only for single material case, it is easy to implement and in many problems one can assume the surrounding gas to be of the same material, thus reducing the problem into single material model.

\section{HIGH PERFORMANCE COMPUTING}

From the perspective of memory volume or arithmetic throughput, the needs of computational scientists are, for all practical purposes, unbounded. As computing power grows, resolution increases, time scales are extended, and the number of phenomena that can be studied together, in context, increases. Application codes have moved from one, to two, to three-dimensional geometries, often on unstructured and dynamically changing grids. In the next generation of application codes there will be a continued drive towards higher fidelity through full multiphysics simulations of real-world phenomena. For WDM, this is particularly important as we strive to model the physics capturing different regimes of material state (solid fragments, molten droplets, vapor and radiating plasma) all on an irregular moving adaptive mesh.

One driving force that is causing applications to undergo a revolutionary change is the underlying change in architecture due to new multicore and heterogenous computing nodes that are currently being designed primarily to increase performance without severe increases in energy consumption. Concurrency will increase exponentially, and neither memory bandwidth nor volume will keep up with the growth in arithmetic performance. Locality must be exploited in a way that allows the memory hierarchy to be used with good power and time efficiency. Soft error rates will increase, and it will no longer be enough to simply drop an occasional checkpoint to achieve resilience. Some of our work on the ALR-AMR is to both exploit these changes to allow for more complicated physics models, while at the same time making sure our simulations run effectively at scale on new architectures.

\section{SUMMARY}

Success at NDCX-II requires an integrated experimental/computational effort. The 3D multi-physics multi-material code, ALE-AMR, is unique in its ability to model hot radiating plasmas and cold fragmenting solids. It is an open science code without export control restrictions that can model NDCX-II. NDCX-II experiments will explore WDM issues such as equation of state effects and heavy ion fusion beam-to-target energy coupling efficiency. Modeling results can be used to plan experiments, critique shots, and compare with experiment data using synthetic diagnostics. We are exploring the addition of surface tension models to the physics of ALE-AMR. Using this new code we hope to explore the process of bubble and droplet formation (two-phase expansion) of superheated metal solids using ion beams. The code has been shown to scale to thousands of CPUs at NERSC, allowing the complex 3D modeling that makes this effort possible. Next generation computing implies an architectural revolution, that with the additional of advanced programming techniques, can further the development of such integrated experimental and computational programs.

We acknowledge the National Energy Research Scientific Computing Center, supported by the Office of Science, U.S. Department of Energy under Contract No. DE-AC02-05CH11231. Work by LBNL under DE-AC0205CH11231 was supported by the Director, Office of Science of the U.S. Department of Energy and the Petascale Initiative in Computational Science and Engineering. Work by LLNL was performed under the auspices of the U.S. Department of Energy by Lawrence Livermore National Security, LLC, Lawrence Livermore National Laboratory under Contract DE-AC52-07NA27344. UCLA and LLNL acknowledge UC Lab Fees Research Grant 09-LR-04116741-BERA.

This work is dedicated to the memory of Frank Bieniosek (1953-2011) who contributed the NDCX-I experimental data images. 


\section{References}

[1] A. Friedman, J. Barnard, R. Cohen, D. Grote, S. Lund, W. Sharp, A. Faltens, E. Henestroza, J. Jung, J. Kwan et al., Phys. Plasmas 17, 056704 (2010)

[2] A.E. Koniges, N.D. Masters, A.C. Fisher, R.W. Anderson, D.C. Eder, T.B. Kaiser, D.S. Bailey, B. Gunney, P. Wang, B. Brown et al., Journal of Physics: Conference Series 244, 032019 (2010)

[3] W. Liu, A.L. Bertozzi, T. Kolokolnikov, Comm. Math. Sci. 10(1), 387 (2012)

[4] B.T. Nadiga, S. Zaleski, Contributions to Mineralogy and Petrology p. 11003 (1995) 